\title{
Probing Transport Theories via Two-Proton Source Imaging
}

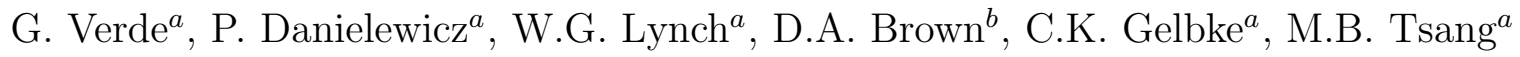 \\ ${ }^{a}$ National Superconducting Cyclotron Laboratory and Department of Physics and Astronomy, \\ Michigan State University, East Lansing, MI 48824, USA \\ ${ }^{b}$ Lawrence Livermore National Laboratory, Livermore, CA 94551-0808, USA
}

(November 2, 2018)

\begin{abstract}
Imaging technique is applied to two-proton correlation functions to extract quantitative information about the space-time properties of the emitting source and about the fraction of protons that can be attributed to fast emission mechanisms. These new analysis techniques resolve important ambiguities that bedeviled prior comparisons between measured correlation functions and those calculated by transport theory. Quantitative comparisons to transport theory are presented here. The results of the present analysis differ from those reported previously for the same reaction systems. The shape of the two-proton emitting sources are strongly sensitive to the details about the in-medium nucleon-nucleon cross sections and their density dependence.
\end{abstract}




\section{INTRODUCTION}

Transport theories have been extensively used to describe the main features of heavy ion collisions at intermediate energies [1-4]. Successful microscopic models have been based on the Boltzmann-Uehling-Uhlenbeck (BUU) equation, which describes the temporal evolution of the one-body phase-space density under the influence of the nuclear mean field and individual nucleon-nucleon collisions [1]. The importance of such models stems from the connections they provide between observables measured in nucleus-nucleus collisions and microscopic quantities like the nuclear mean fields and the in-medium cross section. Recently, this connection has been exploited to place constraints on the equation of state (EOS) of nuclear matter at densities of $2 \rho_{0} \leq \rho \leq 4 \rho_{0}$, where $\rho_{0}$ is the nuclear saturation density [5]. Comparable constraints on the EOS at lower densities require a more detailed understanding of transport phenomena at intermediate energies where the delicate interplay of competing sources of pressure, such as collisions via the residual interaction, govern the collision dynamics [6].

Two-proton correlation functions can provide an important test of transport theory [7-9], through their sensitivity to the space-time properties of nuclear reactions $[10,9,12]$. Initial applications of BUU transport theory to two-proton correlation functions at incident energies of $\mathrm{E} / \mathrm{A}<100 \mathrm{MeV}$ were successful and indicated a significant sensitivity of the calculated correlation functions to the in-medium cross section [7-9]. The application of such techniques to higher incident energies, however, revealed that there were significant problems in reconciling the stronger calculated correlation functions to the weaker measured ones [9]. These problems, discussed below, led to criticisms that BUU transport theory might be an inadequate theoretical tool for such studies, either due to the neglect of the many body correlations in the BUU approach [8] or due to the neglect of the long lived decays of unstable fragments emitted during a collision [9].

In this paper, we largely resolve this issue by showing how more quantitative experimental analyses of two-proton correlations can provide information that can be quantitatively compared to BUU transport theory. We begin by reviewing the basic Koonin-Pratt theoretical approach $[10,13]$ for proton-proton correlation function analyses. We discuss the basic assumptions and limitations of previous analyses that typically assumed a single emission mechanism that could be approximated by one source of radius $r_{0}$ and lifetime $T$ or a dynamical model such as the BUU model and focused upon the height of the correlation function maximum at proton relative momenta of $20 \mathrm{MeV} / \mathrm{c}$. We describe the more quantitative imaging approach that utilizes all the information contained in the shape of the correlation function [14-17]. We apply this approach to two-proton correlation functions measured in central Ar+Sc collisions at $\mathrm{E} / \mathrm{A}=80,120$ and $160 \mathrm{MeV}$ where the original problems were observed [9]. With this technique, we determine the contributions of slow proton emitting sources, allowing direct comparisons to correlations calculated using the BUU equation. We use this information to place constraints on the in-medium nucleon-nucleon cross section.

\section{TWO-PROTON CORRELATION FUNCTIONS}

Experimentally, the (angle-averaged) two-proton correlation function, $1+R(q)$, is defined in terms of the two-particle coincidence yield, $Y\left(\vec{p}_{1}, \vec{p}_{2}\right)$, and the single particle yields $Y\left(\vec{p}_{1}\right)$ and $Y\left(\vec{p}_{2}\right)$ : 


$$
\Sigma Y\left(\vec{p}_{1}, \vec{p}_{2}\right)=k \cdot[1+R(q)] \cdot \Sigma\left[Y\left(\vec{p}_{1}\right) \cdot Y\left(\vec{p}_{2}\right)\right],
$$

Here, $\vec{p}_{1}$ and $\vec{p}_{2}$ are the laboratory momenta of the coincident particles, $q$ is the momentum of relative motion, and $C$ is a normalization constant chosen such that $\langle R(q)\rangle=0$ for large $q$-values where final-state interaction effects are negligible. For a given experimental gating condition, the sums on each sides of Eq. (1) extend over all particle energies and detector combinations corresponding to each bin of $q$.

Eq. 1 is used to build two-proton correlation functions measured in central $\mathrm{Ar}+\mathrm{Sc}$ reactions and shown in Fig. 1. The data points refer to incident energies of $\mathrm{E} / \mathrm{A}=80$ (upper panels), 120 (middle panels) and $160 \mathrm{MeV}$ (lower panels). Experimental details can be found in Ref. [9] and the references therein. The different data symbols refer to proton pairs with total momenta, $P$, in the $\mathrm{CM}$ of the reaction, $200 \leq P \leq 400 \mathrm{MeV} / \mathrm{c}$ (triangles) and $400 \leq P \leq 800 \mathrm{MeV} / \mathrm{c}$ (circles). The data are the same in both the left and right panels.

The shape of these correlation functions reflects the interplay of the short-range nuclear interaction, the antisymmetrization, and the long-range Coulomb interaction between the emitted protons $[10,11]$. The attractive S-wave nuclear interaction leads to the observed pronounced maximum at relative momentum $q \approx 20 \mathrm{MeV} / \mathrm{c}$. At E/A=80 and $120 \mathrm{MeV}$, it is observed that the height of the peak at $20 \mathrm{MeV} / \mathrm{c}$ is larger for proton pairs with larger total momenta in the $\mathrm{CM}$ of the reaction. This total momentum dependence nearly disappears at the highest incident energy, $\mathrm{E} / \mathrm{A}=160 \mathrm{MeV}$.

Theoretically, the correlation function can be calculated using the angle-averaged Koonin-Pratt equation $[10,11]$

$$
R(q)=4 \pi \int d r r^{2} K(q, r) S(r)
$$

The angle-averaged kernel $K(q, r)$ is calculated from the radial part of the antisymmetrical two-proton relative wave-function. At short distances, the kernel is dominated by the strongly attractive single S-wave proton-proton interaction which gives rise to a maximum in $R(q)$ at $20 \mathrm{MeV} / \mathrm{c}$. The long-range repulsive Coulomb interaction produces a minimum at $q \approx 0 \mathrm{MeV} / \mathrm{c}$ to which the antisymmetrization contributes too. The source function $S(r)$ is defined as the probability distribution for emitting a pair of protons with relative distance $r$ at the time the second proton is emitted. The two-particle source function can be determined from

$$
S_{\vec{P}}(r)=\frac{\int d^{3} R \cdot f\left(\frac{1}{2} \vec{P}, \vec{R}+\frac{1}{2} \vec{r}, t_{>}\right) f\left(\frac{1}{2} \vec{P}, \vec{R}-\frac{1}{2} \vec{r}, t_{>}\right)}{\left|\int d^{3} r \cdot f\left(\frac{1}{2} \vec{P}, \vec{r}, t_{>}\right)\right|^{2}}
$$

where $\vec{R}=\frac{1}{2}\left(\vec{r}_{1}+\vec{r}_{2}\right)$ is the center of mass coordinate of the two particles and $\vec{P}=\vec{p}_{1}+\vec{p}_{2}$ is their total momentum. The Wigner function $f\left(\vec{p}, \vec{r}, t_{>}\right)$is the phase-space distribution of particles with momentum $\vec{p}$ and position $\vec{r}$ at some time $t_{>}$after both particles have been emitted. The function $f\left(\vec{p}, \vec{r}, t_{>}\right)$can be expressed in terms of the single particle emission function $d(\vec{p}, \vec{r}, t)$, i.e., the distribution of last emission points for a particle with momentum $\vec{p}$ at location $\vec{r}$ and time $t[13]$ :

$$
f\left(\vec{p}, \vec{r}, t_{>}\right)=\int_{-\infty}^{t_{>}} d t \cdot d\left(\vec{p}, \vec{r}-\vec{p}\left(t_{>}-t\right) / m, t\right)
$$


From Eqs. (2-4), it can be seen that the two-proton correlation function is directly related to the space-time extent of the source that emits the protons. For fast emission where the source spatial dimension exceeds the product of the proton velocity times the source lifetime, the spatial properties dominate the source function. Such is the case for the pre-equilibrium emission predicted by transport models of nuclear reactions where emission timescales are often less than $100 \mathrm{fm} / \mathrm{c}$. For slow emission, on the other hand, the lifetime results in an extended source $[14,18-23, ?, 24]$. Such slow emission processes are characteristic of slow evaporative proton emission from heavy residua and of the decay of excited fragments $A>3$, which are produced abundantly in central collisions, and can decay over very long timescales (of the order of thousands of $\mathrm{fm} / \mathrm{c}$ ).

The sensitivity of correlation functions to such long time scale emissions can be small, especially when the source also contains contributions from short-lived dynamical processes [14]. Long-lived emitting sources contribute to the source function S(r) in Eq. (3) mainly at large relative distance values, $r>10 \mathrm{fm}$, where $K(q, r)$ is dominated by the Coulomb interaction. These large distances influence the correlation function only at very small relative momenta, $q<10 \mathrm{MeV} / \mathrm{c}$, making $1+R(q)$ small where the measurements are difficult to perform $[10,14,25]$. In cases where only long timescale emissions are present, there can also be measurable effects at larger relative momenta $10 \mathrm{MeV} / \mathrm{c} \leq q \leq 20 \mathrm{MeV} / \mathrm{c}$ [24], but these effects are overwhelmed by the contributions to the correlation function maximum at $q=20 \mathrm{MeV} / \mathrm{c}$ from pre-equilibrium emission when such fast emissions are also present [14]. These effects from long-lived decays are so negligible at relative momenta $q>10 \mathrm{MeV} / \mathrm{c}$ that quantitative comparisons to fast emissions predicted by transport theory may therefore be undertaken provided corrections for the long-lived decays are made $[9,14,25]$. In order to understand these corrections we consider the simple limit wherein fast emission provides a fraction $f$ of the emitted protons, $Y_{1, \text { fast }}=f \cdot Y_{1}$, and the rest of the emission with yield, $Y_{1, \text { slow }}=(1-f) \cdot Y_{1}$, is so slow that it contribute negligibly at relative momenta $q>10$ $\mathrm{MeV} / \mathrm{c}$. In this limit, the correlation function $\mathrm{R}(\mathrm{q})$ will reflect only the fast source and will be given by

$$
R(q)=f^{2} \cdot R_{\text {fast }}(q) \equiv \lambda \cdot R_{\text {fast }}(q)
$$

where $R_{\text {fast }}(q)$ denotes the correlation function when only fast emission is present [14]. Equation (5) stipulates that the height of the correlation function peak, $R(20 \mathrm{MeV} / \mathrm{c})$, is attenuated by the factor $\lambda=f^{2}$. Thus, in such limit, the shape of the correlation function peak reflects uniquely the fast two-proton source function and can be used to measure the source shape. The height does not provide unambiguous information about the shape of the fast source, but combined with information obtained from the shape of the correlation function, provides a measure of the relative contribution, $f$, of the fast source [14]. It is this new understanding of two-proton correlations that enables us to remove the ambiguities that bedeviled the previous attempts to relate correlation functions to transport theory.

\section{TWO-PROTON CORRELATIONS IN BUU SIMULATIONS}

The Boltzmann-Uheling-Uhlenbeck (BUU) equation [1] describes the temporal evolution of the one-body density distribution, $f\left(\vec{p}, \vec{r}, t_{>}\right)$: 


$$
\begin{aligned}
\frac{\partial f}{\partial t} & +\frac{\vec{p}}{m} \cdot \vec{\nabla}_{r} f(\vec{p}, \vec{r}, t)-\vec{\nabla}_{r} U(\vec{r}) \cdot \vec{\nabla}_{p} f(\vec{p}, \vec{r}, t) \\
& =\frac{1}{2 \pi^{3} m^{2}} \int d^{3} \vec{q}_{2} \int d \Omega^{\prime} \frac{d \sigma}{d \Omega^{\prime}}\left[\left(1-f_{1}\right)\left(1-f_{2}\right) f_{1}^{\prime} f_{2}^{\prime}+\left(1-f_{1}^{\prime}\right)\left(1-f_{2}^{\prime}\right) f_{1} f_{2}\right]
\end{aligned}
$$

The terms on the l.h.s. of Eq. (6) account for the changes of $f(\vec{p}, \vec{r}, t)$ due to the motion of particles in the average mean field potential, $U(\vec{r})$, produced by other particles. The r.h.s. accounts for changes of $f(\vec{p}, \vec{r}, t)$ due to the nucleon-nucleon collisions. The single particle distribution $f(\vec{p}, \vec{r}, t)$ calculated from BUU can be substituted in Eq. (2-4) to calculate the theoretical two-proton correlation functions for comparison to the experimental data. This approach has been used to test transport theories by comparisons with two-proton correlations [7-9].

One may use such comparisons to constrain the NN collision cross section $d \sigma / d \Omega^{\prime}$ and, possibly, the potential $U(\vec{r})$ in the l.h.s. of Eq. (6). In our calculations we explored different equations of state. Negligible dependence of calculated correlation functions on the equation of state was found at the explored incident energies, in agreement with previous studies $[7,9]$. Therefore we have primarily utilized the stiff EOS with no momentum dependence in the mean fields to minimize computation times. On the other hand, we have observed a significant sensitivity to the in-medium cross section. To explore this sensitivity, we used density dependent in-medium NN cross section of the form [26,27]

$$
\sigma_{i n, \eta}(\rho)=\eta \cdot \rho^{-2 / 3} \cdot \tanh \left[\frac{\sigma_{\text {free }}}{\eta \cdot \rho^{-2 / 3}}\right]
$$

where $\sigma_{\text {free }}$ is the free NN collision cross section and $\rho$ is the surrounding nucleon density. Such parametrization of the density dependence of the in-medium cross sections has been introduced $[26,27]$ to take into account the fact that the geometric cross section radius should not exceed the interparticle distance, $\sigma \lesssim \eta \rho^{-2 / 3}$. Eq. 7 has been shown to be successful in describing stopping observables $[26,27]$. Our simulations were performed for the three different values of $\eta$ listed in Table I. The case of $\eta=\infty$, labelled free, describes simulations with free NN cross sections (Note: $\lim _{\eta \rightarrow \infty} \sigma_{i n, \eta}=\sigma_{\text {free }}$ ). Two choices of reduced in-medium cross sections were used, $\sigma_{i n, 1}(\rho)$ and $\sigma_{i n, 0.6}(\rho)$ corresponding to $\eta=1.0$ and $\eta=0.6$, respectively. As shown in Table I, $\sigma_{i n, 1}(\rho) \approx 0.8 \sigma_{\text {free }}$ and $\sigma_{i n, 0.6}(\rho) \approx 0.6 \sigma_{\text {free }}$ for values of $\sigma_{\text {free }}=30 \mathrm{mb}$ and $\rho=0.17 \mathrm{fm}^{-3}$. On the other hand, $\sigma_{i n, \eta}=\sigma_{\text {free }}$ by construction, when the density is very low, regardless of the value of $\eta$.

The solid lines on the left panels of Fig. 1 show the results of BUU simulations for a stiff equation of state $(\mathrm{K}=380 \mathrm{MeV})$ with no momentum dependent interaction and using a free nucleon-nucleon cross sections $(\eta \rightarrow \infty)$. The dashed and dotted lines on the right panels of Fig. 1 show BUU simulated correlation functions using reduced in-medium cross sections corresponding to $\sigma_{i n, 1}$ and $\sigma_{i n, 0.6}$, respectively. It is evident that this simplest application of BUU is not able to reproduce the variations in the height of the peak at $20 \mathrm{MeV} / \mathrm{c}$ with incident energy and proton total momenta. For example, the correlation functions for high total momenta proton pairs, $400 \leq P \leq 800 \mathrm{MeV} / \mathrm{c}$, are systematically overpredicted by the BUU model. Discrepancies of this magnitude cannot be remedied by making alternative choices for the EOS, by using momentum-dependent interactions, or by making reasonable modifications of the in-medium nucleon-nucleon cross section. 
When such discrepancies were observed in refs. [9], they were attributed to the influence of the statistical decay of fragments and other long-lived particle emitting sources. Since the BUU model has no realistic prescription for complex fragment formation, quantitative comparisons between BUU predictions and experimental data require corrections taking into account properly the contributions from such long lifetime emissions. Previous attempts to apply such corrections could provide only a qualitative description of measured correlations [9]. BUU calculations were adjusted comparing the peak values of the calculated and measured correlation functions at $q=20 \mathrm{MeV}$ and assuming the long-lived components to be represented as a single lifetime secondary decay contributions. This approach did not allow an independent test of BUU and no insights into nuclear transport properties could be extracted. As we show below, we will accomplish both by taking into account all the information contained in the shape of the correlation function.

\section{IMAGING ANALYSIS OF TWO-PROTON CORRELATIONS}

We study the correlation function data displayed in Fig. 1 using the imaging analysis technique introduced in [15-17]. The analysis is based on the extraction of the source function $S(r)$ by inverting the integral equation Eq. (3) using the measured correlation function $1+R(q)$. Thus, "imaging the source" means inverting this equation. Such an inversion is not completely straightforward because one has to take into account experimental factors (limited statistics, finite widths of momentum bins, etc.) and the intrinsic resolution of the kernel $K(q, r)$. Small fluctuations in the data, even if well within statistical or systematic errors, can lead to large changes in the imaged source function. On the other hand, successful inversion of the correlation function has the virtue of being model independent.

The source functions $S(r)$ in our imaging analyses are expanded in a superposition of polynomial splines [15],

$$
S(r)=\sum_{j} S_{j} \cdot B_{j}(r)
$$

By using this expansion, the Koonin-Pratt equation is discretized into a matrix equation

$$
C_{i}^{t h}=1+R_{i}^{t h}=\sum_{j} K_{i j} \cdot S_{j}
$$

where $K_{i j}$ can be calculated from

$$
K_{i j}=\int d r \cdot K(q, r) B_{j}(r)
$$

We introduce the superscript "th" on C to distinguish theoretical correlation functions, determined by Eq. (1) from experimental correlation functions that we label with superscript "ex", $C_{i}^{e x}=1+R_{i}^{e x}$.

The unknown coefficients, $S_{j}$, in the matrix equation (9), are determined by minimizing the value of the $\chi^{2}$ between the experimental correlation function and the one calculated from Eq. (9):

$$
\chi^{2}=\sum_{i}\left(C_{i}^{e x}-\sum_{j} k_{i j} S_{j}\right)^{2} / \Delta^{2} C_{i}^{e x}
$$


The conditions of the minimum can be inverted to yield an algebraic expression for the values of $S_{j}$ :

$$
S_{j}=\sum_{i}\left[\left(K_{T}\left(\Delta^{2} C^{e x}\right)^{-1} K\right)^{-1} K^{T} B\right]_{j i}\left(C_{i}^{e x}-1\right)
$$

where $K^{T}$ is the transpose of the kernel matrix given by Eq. (10). The uncertainties in the source coefficients, $\Delta S$, are given by the square root of the diagonal elements of the covariance matrix of the source

$$
\Delta^{2} S=\left[K^{T}\left(\Delta^{2} C\right)^{-1} K\right]^{-1}
$$

Constraints may be included in the inversion using well defined procedures, as described in Refs. [15-17]. Using this formalism, we analyzed the data shown in Fig. 1 by decomposing the source in a superposition of six spline polynomials of the 3rd order over the interval $0 \leq r \leq 18 \mathrm{fm}$, as described in Refs. [15] and [14]. The grey areas and the hatched areas in Fig. 2 show the results of this imaging analysis for total proton momenta $400 \leq P \leq 800$ and $200 \leq P \leq 400$, respectively. The extracted source functions are shown in Figs. 4-6 for $\mathrm{E} / \mathrm{A}=80,120$ and $160 \mathrm{MeV}$, respectively. The same grey and hatched areas as in Fig. 2 have been used to represent the imaged sources. In Fig. 4-6, the top panel refers to the $400 \leq P \leq 800 \mathrm{MeV} / \mathrm{c}$ gate, while the bottom panel refers to the proton pairs with $200 \leq P \leq$ $400 \mathrm{MeV} / \mathrm{c}$.

Since the imaging analysis reproduces the whole shape of the correlation functions, the extracted sources, shown in Fig. 4-6, provide a measure of the whole information content that can be extracted from two-proton correlation functions. The analyses shown in Fig. 4-6 provide significant information only for the short-range portion of the real two-proton emitting sources, dominated by early dynamical emissions. The $r_{1 / 2}$-values of these profiles are listed in Table II and represent an estimate of the space-time extent of these sources. Long lifetime decays dominate mainly the tail of the source at $r \geq 10 \mathrm{fm}$. These cannot be imaged in detail because of the poor resolution of the data at small relative momenta $(q<10 \mathrm{MeV} / \mathrm{c})$. Consequently, from the square root of the integral of these profiles, one can estimate the fractional amount of protons emitted by the fast dynamical source, $f_{d y n}$, by using the equation:

$$
f_{d y n}=\sqrt{\int_{0}^{r_{\max }} d^{3} r \cdot S(r)}
$$

One should keep in mind that Eq. (14) gives the fraction of the proton pairs that are emitted with $r \leq r_{\max }$, and as such is sensitive to the value of $r_{\max }$. This is shown in Table II where the $f_{d y n}$ values obtained for $r_{\max }=2 \cdot r_{1} / 2, r_{\max }=2.5 \cdot r_{1} / 2$ and $r_{\max }=3 \cdot r_{1} / 2$ are also listed. This sensitivity issue does not influence comparisons with BUU, because the BUU does not provide accurate predictions at large values of $r$ anyway. The imaging analysis does provide direct measurements of the shape of the pre-equilibrium source at $r \leq r_{\max }$ and this can be compared directly to BUU. Only the knowledge of how to effectively remove this sensitivity to long time scale decay can allow quantitative comparisons between transport theory and two-proton correlation functions. Such task will now be accomplished for the 
first time. We use the imaging technique to perform such comparisons and extract the information contained in correlation functions regarding the transport of nucleons during the early stages of the collision.

\section{CONSTRAINING THE FRACTION OF LONG LIFETIME EMISSIONS FOR BUU SOURCES}

The comparison of BUU simulations to experimental data can be performed in two different ways. One can directly compare calculated and measured correlation functions as attempted in Figure 1, but now one can properly correct for the contributions of long timescale decays. Alternatively, one can directly compare the two-proton sources calculated using BUU and Eq. (4) to the source profiles extracted from the data with the imaging technique. We find the latter to be the more effective approach.

The key to either comparison is the correction for the influence of long-lived decays. Essentially, the physics of these very long-lived decays is contained in the parameter $\lambda$, which defines the fraction of the source function that is fast and can, in principle, be described by transport theory. Fig. 3 demonstrates the problem. The dashed line in the figure shows the BUU source function, which is normalized so that $4 \pi \int r^{2} d r S(r)=1$, according to its definition in Eq. 4. The grey area is the experimentally determined source function. It is normalized so that $4 \pi \int r^{2} d r S(r)=1$. However, the experimental source must be smaller at $\mathrm{r}<16 \mathrm{fm}$ because its normalization also reflects the contributions at larger $\mathrm{r}$ from long timescale decays. To make the BUU sources comparable to those of the data, it is therefore necessary to likewise renormalize the BUU source, requiring its normalization to reflect only the fraction $\lambda$ of the total source that the BUU calculations can reproduce.

To proceed further, it is imperative to correct the normalization $\lambda$ of the BUU source function to reflect the measured relative contributions of short-lived decays. The most accurate normalization appears to be obtained by requiring that the normalized source, derived from BUU

$$
S_{d y n}^{B U U}(r)=\lambda \cdot S^{B U U}(r)
$$

to most accurately replicate the measured source function by adjusting $\lambda$ to minimize the quantity:

$$
\chi^{2}=\sum_{i} \frac{\left[S^{I m g}\left(r_{i}\right)-\lambda \cdot S_{d y n}^{B U U}\left(r_{i}\right)\right]^{2}}{\left[\Delta S^{I m g}\left(r_{i}\right)\right]^{2}+\left[\lambda \cdot \Delta S^{B U U}\left(r_{i}\right)\right]^{2}}
$$

Here, $S^{I m g}(r)$ represents the imaged source, and the uncertainties, $\Delta S^{\operatorname{Img}}(r)$, are determined using Eq. (13). The uncertainties of the BUU sources, $\triangle S^{B U U}(r)$, are determined from the statistics of the simulations. The sum in Eq. (16) is extended over the region of relative distances $r$ where the imaged source function is still significant $(\approx 12 \mathrm{fm})$. The BUU source, after being properly renormalized using $\mathrm{Eq}(15)$ with $\lambda=0.52$, is represented in Fig. 3 by the solid line. Clearly, one could have also obtained similar results by requiring equality between BUU and measured values for the integral, however, the values for this integral are extremely sensitive to uncertainties in the measured values for $S(r)$ at large radii. We find the normalization procedure of Eq. 15 to be more reliable when such uncertainties are taken into consideration. 
This technique has been extended to all incident energies and $P$ gates, as shown in Fig. 4-6. The imaged sources are represented by the grey (upper panel, $\mathrm{P}=400-800 \mathrm{MeV} / \mathrm{c}$ ) and the hatched (lower panel, $\mathrm{P}=200-400 \mathrm{meV} / \mathrm{c}$ ) areas. The solid, dashed and dotted lines correspond to two-proton BUU sources for free, $\sigma_{i n, 1}$ and $\sigma_{i n, 0.6} \mathrm{NN}$ cross sections, respectively. At all incident energies, high total momentum two-proton sources are well described by BUU simulations. Furthermore, for such energetic protons, two-particle sources do not depend on the choice of the N-N cross section. At low total momenta, however, the two-proton source functions display a very strong sensitivity to details about the in-medium N-N cross section (see lower panels in Fig. 4-6). This sensitivity is further explored in the following section.

Before going on to the investigation of the in-medium N-N cross section, we demonstrate in Figs. 7-9 that the renormalization of the BUU source functions described above resolves most of the discrepancies between measured and BUU correlation functions shown in Fig. 1. Figure 7 shows the comparison between measured and calculated correlation functions in collisions at $\mathrm{E} / \mathrm{A}=80 \mathrm{MeV}$. The left (right) panel of the figure refers to the gate $400 \leq P \leq$ $800 \mathrm{MeV} / \mathrm{c}(200 \leq P \leq 400 \mathrm{MeV} / \mathrm{c})$. The solid and dashed lines correspond to the free and $\sigma_{i n, 0.6} \mathrm{NN}$ cross sections, respectively. The same conventions have been used in Fig. 8 $(\mathrm{E} / \mathrm{A}=120 \mathrm{MeV})$ and Fig. $9(\mathrm{E} / \mathrm{A}=160 \mathrm{MeV})$. As already stated, a comparison between the shape of the emitting sources directly corresponds to a comparison between the shape of the correlation peaks. Thus, it is not surprising to see that, at high total momenta, $400 \leq P \leq 800$ $\mathrm{MeV} / \mathrm{c}$ (see left panels on Fig. 7-9), BUU simulations can provide a reasonable description of the correlation functions; this result could already be anticipated from Fig. 4-6 (upper panels). At low total momenta $(200 \leq P \leq 400 \mathrm{MeV} / \mathrm{c})$, the shape of the BUU correlations becomes very sensitive to the details of the N-N cross section. Therefore, it is in this low total momentum gate that two-proton correlation analysis can provide sensitive probes of transport models of heavy-ion collisions, provided that attention is paid to the whole shape of the correlation functions.

\section{PROBING THE NUCLEON-NUCLEON COLLISION CROSS SECTION}

The overall features of Fig. 4-6 show that for free NN cross sections, the BUU predicts two-particle sources that are too extended. Correspondingly, they produce two-proton correlation peaks (Fig. 7-9), which are too narrow. This correspondence between the size of the source and the width of the correlation peak was already extensively illustrated in Ref. [14], where a linear relation between these two quantities was derived. At $\mathrm{E} / \mathrm{A}=80$ and $120 \mathrm{MeV}$, a reduction of the in-medium NN cross section in BUU simulations produces sources that are in reasonable agreement with the imaged ones. We note that this conclusion is different from the one reached in Ref. [7]. There, the best agreement between BUU calculations and correlation function data was attained when free cross section values were used. Our conclusions indicate that the previous results were reached because the influence of long-lived decays were not properly taken into account by the analysis of Ref. [7]. The results on the cross section reductions reported here are consistent with the conclusions deduced in Ref. [28] from the analysis of flow data.

Despite these successes, at higher energies, $\mathrm{E} / \mathrm{A}=160 \mathrm{MeV}$, the shape of the low total momentum two-proton source from imaging exhibits a core+tail behavior that is not repro- 
duced by transport model simulations. The shape of the BUU two-proton source appears incorrect, no matter what cross section is used. However, it should be mentioned that at $\mathrm{E} / \mathrm{A}=160 \mathrm{MeV}$, for $200 \leq P \leq 400 \mathrm{MeV} / \mathrm{c}$ the correlation function cannot be easily normalized to 1 as in the other cases studied in this work. The overall shape of the correlation, if extended to larger relative momenta, suggests the presence of space- momentum correlations that may be due to collective motion. Such kind of correlations are different than those described by the Koonin-Pratt formalism, and require special study that is beyond the scope of this article. We therefore refrain, at present, from drawing definitive conclusions about the disagreements in the low momentum gate $200 \leq P \leq 400 \mathrm{MeV} / \mathrm{c}$ observed in Fig. 6 and 9 .

The reduction in the size of the source function when the in-medium cross section is reduced can be simply understood. The two-proton sources calculated from Eq. (3) and (4) correspond to the distribution of protons when the second proton is emitted, i.e. at the moment of its last collision with another nucleon. As a consequence, these sources largely reflect properties of the phase-space distribution of particles at freeze-out when the nucleon density is low and collisions cease. This is particularly true when one selects low total momentum proton pairs in a range of $200 \leq P \leq 400 \mathrm{MeV} / \mathrm{c}$. On average, such low momentum pairs decouple from the system at a later time when the system has expanded further than for the high momentum pairs. We assume schematically that the low density freeze-out stage is achieved when the distance that a nucleon travels before colliding with another nucleon, $d$, is of the order of the entire spatial extent $R$ of the system . The nucleon mean free path, $d$, can be written as $d=1 / \rho \sigma$, where $\rho=A /\left(4 \pi R^{3} / 3\right)$ is the nucleon density and $\sigma$ is the N-N cross section. It follows that one should expect a sensitivity of the spatial extent of the source to the N-N cross section, of the form $R \propto \sqrt{\sigma \cdot A}$. This last relation explains why the size of the two-proton source function decreases for reduced N-N cross sections, like $\sigma_{i n, 1}$ and $\sigma_{i n, 0.6}$. The reasoning needs to be modified, if a strong collective motion develops in the system. In this case, the size of the sources becomes smaller than the entire spatial extent of the system, but it can be still shown that the direct dependence on cross section persists, with source size $\propto \sqrt{\sigma \cdot A}$.

In order to confirm such qualitative arguments, we performed additional reaction simulations reducing the cross section alternatively when the mean local nucleon density, $\rho$, is lower or higher that $0.5 \rho_{0}$ (using $\rho_{0}=0.16 \mathrm{fm}^{-3}$ as the nucleon saturation density). The solid and dashed lines in Figure 10 show the simulated temporal evolution of the central and maximal $\rho / \rho_{0}$, respectively, during a $\mathrm{Ar}+\mathrm{Sc}$ central collision at $\mathrm{E} / \mathrm{A}=120 \mathrm{MeV}$. (The condition of $\rho / \rho_{0}<0.5$ favors later stages of the reaction prior to the global freeze-out.) The hatched area in Fig. 11 corresponds to the imaged source for $\mathrm{E} / \mathrm{A}=120 \mathrm{MeV}$ and total momenta $200 \leq P \leq 400 \mathrm{MeV} / \mathrm{c}$. The dashed line represents the BUU two-proton source when we use a reduced $\sigma_{i n, 0.6} \mathrm{NN}$ cross-section only in the higher density region, $\rho / \rho_{0}>0.5$, and a free $\mathrm{NN}$ cross section at lower densities, $\rho / \rho_{0}<0.5$. The dot-dashed line refers to the opposite situation, where a free NN cross section is used for $\rho / \rho_{0}>0.5$, and $\sigma_{i n, 0.6}$ is used at lower densities. The dotted and solid lines follow the same conventions of Fig. 4-6 and correspond to sources obtained using $\sigma_{\text {free }}$ and $\sigma_{i n, 0.6}$ at all densities, respectively. It appears clearly that, by reducing the cross section only at low density, BUU generates nearly the same two-proton source as is generated when the cross section is reduced at all densities. These results show that two-proton correlation functions are sensitive to the details of the 
NN collision cross sections at densities less than $\rho_{0} / 2$. According to the results of the BUU predictions shown in Fig. 10, such low densities are typically reached during the later stages of the reaction when the system approaches freeze-out.

\section{SUMMARY AND CONCLUSIONS}

In this paper we have compared two-proton correlation functions measured in central $\mathrm{Ar}+\mathrm{Sc}$ collisions at $\mathrm{E} / \mathrm{A}=80,120$ and $160 \mathrm{MeV}$ to $\mathrm{BUU}$ simulations with different choices of the NN collision cross section. The observed discrepancies in the height of the peak at $20 \mathrm{MeV} / \mathrm{c}$ have been discussed in the context of the deficiencies of transport theories in dealing with long-lived emitting sources due to secondary decays of unstable fragments. The imaging technique provides the tools to correct for such deficiencies of the model. Indeed, this detailed shape analysis of the correlation peak constrains the contributions from long- lived secondary decay emissions without the need to invoke any additional BUU model assumptions. Using these constraints, the previously observed discrepancies between the peak values of BUU and measured correlation functions are removed and quantitative comparisons between theory and experiment can be made. However, the most important consequence of the renormalization of the BUU sources functions is that the shape of the calculated two-proton sources in BUU can be directly compared to the shape of the imaged sources.

Our results show that the shape of two-proton correlation functions is very sensitive to the choice of the NN collision cross sections in BUU simulations especially at low density. This strong sensitivity allowed us to conclude that reduced in-medium cross-sections are experimentally preferred. This result is consistent with the conclusions obtained from transverse flow analysis [28]. We performed an analysis of the density dependence of such sensitivity to the NN cross section. This analysis showed that the sensitivity the in-medium cross section is somewhat stronger at the low densities, confirming that the sensitivity of the correlation function to the cross-section stems from the fact that the cross section strongly influences the freeze-out volume.

In conclusion, this study shows that detailed imaging analyses can provide the information needed for quantitative tests of transport theories of heavy-ion collisions. Further investigations with these techniques offer exciting opportunities to the influence of collective motion on two particle correlations and to utilize the sensitivity of two-proton correlations to further explore the freeze-out stages of the reaction.

The National Science Foundation under Grant Nos. PHY-95-28844 and PHY-0070818 has supported this work.

This work was performed under the auspices of the U.S. Department of Energy by University of California, Lawrence Livermore National Laboratory under Contract W-7405-Eng-48. 


\section{REFERENCES}

[1] G.F. Bertsch and S. Das Gupta, Phys. Rep. 160, 189 (1988) and refs. therein.

[2] J. Aichelin, Phys. Rep. 202, 233 (1991) and refs. therein.

[3] A. Ono, H. Horiuchi, T. Maruyama, and A. Ohnishi, Phys. Rev. Lett. 68, 2898 (1992).

[4] A. Ono and H. Horiuchi, Phys. Rev. C53, 2958 (1996).

[5] P. Danielewicz, R. Lacey, and W. G. Lynch, LANL nucl-th/0208016.

[6] D. J. Magestro, W. Bauer, and G. D. Westfall, Phys. Rev. C 62, 041603(R) (2000).

[7] W. G. Gong, W. Bauer, C. K. Gelbke, N. Carlin, R. T. de Souza, Y. D. Kim, W. G. Lynch, T. Murakami, G. Poggi, D. P. Sanderson, M. B. Tsang, H. M. Xu, S. Pratt, D. E. Fields, K. Kwiatkowski, R. Planeta, V. E. Viola, Jr., and S. J. Yennello, Phys. Rev. Lett. 65, 2114 (1990).

[8] G. J. Kunde, J. Pochodzalla, E. Berdermann, B. Berthier, C. Cerruti, C. K. Gelbke, J. Hubele, P. Kreutz, S. Leray, R. Lucas, U. Lynen, U. Milkau, C. Ng, C. H. Pinkenburg, G. Raciti, H. Sann, and W. Trautmann, Phys. Rev. Lett. 70, 2545 (1993).

[9] D. O. Handzy, W. Bauer, F. C. Daffin, S. J. Gaff, C. K. Gelbke, T. Glasmacher, E. Gualtieri, S. Hannuschke, M. J. Huang, G. J. Kunde, R. Lacey, T. Li, M. A. Lisa, W. J. Llope, W. G. Lynch, L. Martin, C. P. Montoya, R. Pak, G. F. Peaslee, S. Pratt, C. Schwarz, N. Stone, M. B. Tsang, A. M. Vander Molen, G. D. Westfall, J. Yee, and S. J. Yennello, Phys. Rev. Lett. 75, 2916 (1995).

[10] S.E. Koonin, Phys. Lett. 70 B, 43 (1977).

[11] D.H. Boal, C.K. Gelbke and B.K. Jennings, Rev. Mod, Phys. 62, 553 (1990) and references therein.

[12] U. Heinz and B.V. Jacak, Annu. Rev. Nucl. Part. Sci. 49, 529 (1999).

[13] W. G. Gong, W. Bauer, and C. K. Gelbke, Phys. Rev. C 43, 781 (1991).

[14] G. Verde, D. A. Brown, P. Danielewicz, C. K. Gelbke, W. G. Lynch, and M. B. Tsang, Phys. rev. C 65, 054609 (2002).

[15] D.A. Brown and P. Danielewicz, Phys. Rev. C 64, 104902 (2001).

[16] D.A. Brown and P. Danielewicz, Phys. Lett. B 398, 252 (1997).

[17] D.A. Brown and P. Danielewicz, Phys. Rev. C 57, 2474 (1998).

[18] W.G. Gong, Y. D. Kim, and C. K. Gelbke, Phys. Rev. C 45, 863 (1992).

[19] P. A. DeYoung, M. S. Gordon, Xiu qin Lu, R. L. McGrath, J. M. Alexander, D. M. de Castro Rizzo, and L. C. Vaz, Phys. Rev. C 39, 128 (1989).

[20] P. A. De Young, C. J. Gelderloos, D. Kortering, J. Sarafa, K. Zienert, M. S. Gordon, B. J. Fineman, G. P. Gilfoyle, X. Lu, R. L. McGrath, D. M. de Castro Rizzo, J. M. Alexander, G. Auger, S. Kox, L. C. Vaz, C. Beck, D. J. Henderson, D. G. Kovar and M. F. Vineyard, Phys. Rev. C 41, R1885 (1990).

[21] W. G. Gong, C. K. Gelbke, N. Carlin, R. T. de Souza, Y. D. Kim, W. G. Lynch, T. Murakami, G. Poggi, D. Sanderson, M. B. Tsang, H. M. Xu, D. E. Fields, K. Kawiatkowski, R. Pl-strokeaneta, V. E. Viola, Jr., S. J. Yennello and S. Pratt, Phys. Lett. B 246, 21 (1990).

[22] D. Goujdami, F. Guilbault, C. Lebrun, D. Ardouin, H. Dabrowski, S. Pratt, P. Lautridou, R. Boisgard, J. Qubert and A. Pghaire, Z. Phys. A 339, 293 (1991)

[23] A. Elmaani, N. N. Ajitanand, J. M. Alexander, R. Lacey, S. Kox, E. Liatard, F. Merchez, T. Motobayashi, B. Noren, C. Perrin, D. Rebreyend, Tsan Ung Chan, G. Auger and S. Goult, Phys. Rev. C 43, R2474 (1991). 
[24] M. A. Lisa, W. G. Gong, C. K. Gelbke, N. Carlin, R. T. de Souza, Y. D. Kim, W. G. Lynch, T. Murakami, G. Poggi, M. B. Tsang, and H. M. Xu, K. Kwiatkowski, V. E. Viola, Jr., and S. J. Yennello, Phys. Rev. C49, 2788 (1994).

[25] G.J. Kunde, Proceedings. of CORINNE 90, World Scientific (1990), p.125

[26] P. Danielewicz, Nucl. Phys. A673, 375 (2000).

[27] P. Danielewicz, Acta Phys. Pol. B 33, 45 (2002).

[28] G.D. Westfall, W. Bauer, D. Craig, M. Cronqvist, E. Gaultieri, S. Hannuschke, D. Klakow, T. Li, T. Reposeur, A.M. Vander Molen, W.K. Wilson, J.S. Winfield, J. Yee, S.J. Yennello, R. Lacey, A. Elmaani, J. Lauret, A. Nadasen and E. Norbeck, Phys. Rev. Lett. 71, 1986 (1993). 


\section{TABLES}

TABLE I. Used in-medium cross section (see Eq. (7)). The last column shows the reduction of the in-medium cross section, for the exemplary $\sigma_{\text {free }}=30 \mathrm{mb}$, at normal nuclear density.

\begin{tabular}{ccc}
\hline \hline & $\eta$ & $\begin{array}{c}\sigma_{\text {in }- \text { med }} / \sigma_{\text {free }} \\
\left(\rho=\rho_{0}\right)\end{array}$ \\
\hline free & & 1 \\
$\sigma_{i n, 1}$ & $\infty$ & 0.8 \\
$\sigma_{i n, 0.6}$ & 1.0 & 0.6 \\
\hline \hline
\end{tabular}

TABLE II. $r_{1 / 2}$ and $f$ values of the imaged sources. The $f$ values are evaluated using Eq. (14) with $r_{\max }=2 \cdot r_{1 / 2}, r_{\max }=2.5 \cdot r_{1 / 2}$ and $r_{\max }=3 \cdot r_{1 / 2}$. See text for more details.

\begin{tabular}{cccccc}
\hline \hline $\mathrm{E} / \mathrm{A}(\mathrm{MeV})$ & $\mathrm{P}(\mathrm{MeV} / \mathrm{c})$ & $\mathrm{r}_{1 / 2}(f m)$ & $\mathrm{f}\left(\mathrm{r}_{\max }=2 \mathrm{r}_{1 / 2}\right)$ & $\mathrm{f}\left(\mathrm{r}_{\max }=2.5 \mathrm{r}_{1 / 2}\right)$ & $\mathrm{f}_{\left(\mathrm{r}_{\max }=3 \mathrm{r}_{1 / 2}\right)}$ \\
\hline 80 & $200-400$ & 3.76 & 0.52 & 0.59 & 0.64 \\
80 & $400-800$ & 3.55 & 0.62 & 0.69 & 0.73 \\
\hline 120 & $200-400$ & 3.18 & 0.44 & 0.49 & 0.54 \\
120 & $400-800$ & 3.46 & 0.59 & 0.67 & 0.73 \\
\hline 160 & $200-400$ & 2.88 & 0.48 & 0.51 & 0.54 \\
160 & $400-800$ & 3.38 & 0.59 & 0.66 & 0.71 \\
\hline \hline
\end{tabular}




\section{FIGURE CAPTIONS:}

Figure 1. Two-proton correlation functions in Ar+Sc central collisions at $\mathrm{E} / \mathrm{A}=80 \mathrm{MeV}$ (upper panels), $120 \mathrm{MeV}$ (central panels) and $160 \mathrm{MeV}$ (bottom panels). The different data symbols refer to two gates in total momenta, $\mathrm{P}$, of the proton pairs in the $\mathrm{CM}$ of the reaction: $200 \leq P \leq 400 \mathrm{MeV} / \mathrm{c}$ (triangles), $400 \leq P \leq 800 \mathrm{MeV} / \mathrm{c}$ (circles). The data are reproduced in the left and right panels. The lines correspond to BUU simulations. The continuous lines correspond to simulations with free NN cross sections. The dashed and dotted lines correspond to reduced in-medium cross sections with $\eta=1.0$ and $\eta=0.6$, respectively.

Figure 2. Two-proton correlation functions in Ar+Sc central collisions at $\mathrm{E} / \mathrm{A}=80 \mathrm{MeV}$ (upper panels), $120 \mathrm{MeV}$ (central panels) and $160 \mathrm{MeV}$ (bottom panels). Triangles: proton pairs with total momenta $200 \leq P \leq 400 \mathrm{MeV} / \mathrm{c}$. Circles: proton pairs with total momenta $400 \leq P \leq 800 \mathrm{MeV} / \mathrm{c}$. The grey and hatched areas show the results of the imaging analysis of the experimental data.

Figure 3. Renormalization of BUU two-proton sources, as described in Eq. 9 and 10. The grey area refers to the imaged source for $\mathrm{Ar}+\mathrm{Sc}$ reactions at $\mathrm{E} / \mathrm{A}=80 \mathrm{MeV}$ and for high total momenta, $400 \leq P \leq 800 \mathrm{MeV} / \mathrm{c}$. The thin dashed and solid lines represent the BUU sources before and after renormalization, respectively.

Figure 4. The grey (upper panel) and hatched (lower panel) areas are the two-proton sources extracted from the imaging analysis shown in Fig. 2 for Ar+Sc central collisions at $\mathrm{E} / \mathrm{A}=80 \mathrm{MeV}$. The upper (lower) panel refers to a total momentum gate of $400 \leq P \leq 800$ $\mathrm{MeV} / \mathrm{c}(200 \leq P \leq 400 \mathrm{MeV} / \mathrm{c})$. The thin solid, dotted and dashed lines represent the renormalized BUU sources using free, $\sigma_{i n, 1}$ and $\sigma_{i n, 0.6} \mathrm{NN}$ collision cross sections, respectively.

Figure 5. The grey (upper panel) and hatched (lower panel) areas are the two-proton sources extracted from the imaging analysis shown in Fig. 2 for $\mathrm{Ar}+\mathrm{Sc}$ central collisions at $\mathrm{E} / \mathrm{A}=120 \mathrm{MeV}$. The upper (lower) panel refers to a total momentum gate of $400 \leq P \leq 800$ $\mathrm{MeV} / \mathrm{c}(200 \leq P \leq 400 \mathrm{MeV} / \mathrm{c})$. The thin solid, dotted and dashed lines represent the renormalized BUU sources using free, $\sigma_{i n, 1}$ and $\sigma_{i n, 0.6} \mathrm{NN}$ collision cross sections, respectively.

Figure 6. The grey (upper panel) and hatched (lower panel) areas are the two-proton sources extracted from the imaging analysis shown in Fig. 2 for Ar+Sc central collisions at $\mathrm{E} / \mathrm{A}=160 \mathrm{MeV}$. The upper (lower) panel refers to a total momentum gate of $400 \leq P \leq 800$ $\mathrm{MeV} / \mathrm{c}(200 \leq P \leq 400 \mathrm{MeV} / \mathrm{c})$. The thin solid, dotted and dashed lines represent the renormalized BUU sources using free, $\sigma_{i n, 1}$ and $\sigma_{i n, 0.6} \mathrm{NN}$ collision cross sections, respectively.

Figure 7. The data points show measured two-proton correlations in central $\mathrm{Ar}+\mathrm{Sc}$ reactions at $\mathrm{E} / \mathrm{A}=80 \mathrm{MeV}$. The left (right) panel corresponds to a total momentum gate $400 \leq P \leq 800 \mathrm{MeV} / \mathrm{c}(200 \leq P \leq 400 \mathrm{MeV} / \mathrm{c})$. The thin solid and dashed lines refer to BUU correlation functions after the BUU two-proton sources have been renormalized as described in the text and in Fig. 3.

Figure 8. The data points show measured two-proton correlations in central $\mathrm{Ar}+\mathrm{Sc}$ reactions at $\mathrm{E} / \mathrm{A}=120 \mathrm{MeV}$. The left (right) panel corresponds to a total momentum gate $400 \leq P \leq 800 \mathrm{MeV} / \mathrm{c}(200 \leq P \leq 400 \mathrm{MeV} / \mathrm{c})$. The thin solid and dashed lines refer to BUU correlation functions after the BUU two-proton sources have been renormalized as described in the text and in Fig. 3.

Figure 9. The data points show measured two-proton correlations in central Ar+Sc reactions at $\mathrm{E} / \mathrm{A}=160 \mathrm{MeV}$. The left (right) panel corresponds to a total momentum gate $400 \leq P \leq 800 \mathrm{MeV} / \mathrm{c}(200 \leq P \leq 400 \mathrm{MeV} / \mathrm{c})$. The thin solid and dashed lines refer to BUU 
correlation functions after the BUU two-proton sources have been renormalized as described in the text and in Fig. 3.

Figure 10. Central (solid line) and maximal (dashed line) nuclear matter density $\rho$, normalized to saturation density, $\rho_{0}(=0.17 \mathrm{fm}-3)$, as a function of time, $t$, during a central $\mathrm{Ar}+\mathrm{Sc}$ collision at $\mathrm{E} / \mathrm{A}=120 \mathrm{MeV}$, as simulated by the BUU model.

Figure 11. Hatched area: two-proton source function extracted from imaging analysis of $\mathrm{Ar}+\mathrm{Sc}$ collisions at $\mathrm{E} / \mathrm{A}=120 \mathrm{MeV}$ and for low total momentum proton pairs $(200 \leq P \leq 400$ $\mathrm{MeV} / \mathrm{c}$ ). The solid and dotted lines correspond to two-proton sources simulated by BUU, after renormalization, and using free and $\sigma_{i n, 0.6} \mathrm{NN}$ cross sections, respectively. The dashed and dot-dashed line refer to simulations where NN cross sections have been reduced only at higher densities $\left(\rho / \rho_{0}>0.5\right)$ or only at lower densities $\left(\rho / \rho_{0}<0.5\right)$, respectively. 
This figure "fig1_los.gif" is available in "gif" format from: http://arxiv.org/ps/nucl-ex/0301013v1 
This figure "fig2_los.gif" is available in "gif" format from: http://arxiv.org/ps/nucl-ex/0301013v1 
This figure "fig3_los.gif" is available in "gif" format from: http://arxiv.org/ps/nucl-ex/0301013v1 
This figure "fig4_los.gif" is available in "gif" format from: http://arxiv.org/ps/nucl-ex/0301013v1 
This figure "fig5_los.gif" is available in "gif" format from: http://arxiv.org/ps/nucl-ex/0301013v1 
This figure "fig6_los.gif" is available in "gif" format from: http://arxiv.org/ps/nucl-ex/0301013v1 
This figure "fig7_los.gif" is available in "gif" format from: http://arxiv.org/ps/nucl-ex/0301013v1 
This figure "fig8_los.gif" is available in "gif" format from: http://arxiv.org/ps/nucl-ex/0301013v1 
This figure "fig9_los.gif" is available in "gif" format from: http://arxiv.org/ps/nucl-ex/0301013v1 
This figure "fig10_los.gif" is available in "gif" format from: http://arxiv.org/ps/nucl-ex/0301013v1 
This figure "fig11_los.gif" is available in "gif" format from: http://arxiv.org/ps/nucl-ex/0301013v1 